PROCEEDINGS OF THE AMERICAN MATHEMATICAL SOCIETY

Volume 125, Number 7, July 1997, Pages 2021-2027

S $0002-9939(97) 03773-8$

\title{
FIXED POINT PROPERTY AND NORMAL STRUCTURE FOR BANACH SPACES ASSOCIATED TO LOCALLY COMPACT GROUPS
}

\author{
ANTHONY TO-MING LAU, PETER F. MAH, AND ALI ÜLGER
}

(Communicated by J. Marshall Ash)

\begin{abstract}
In this paper we investigate when various Banach spaces associated to a locally compact group $G$ have the fixed point property for nonexpansive mappings or normal structure. We give sufficient conditions and some necessary conditions about $G$ for the Fourier and Fourier-Stieltjes algebras to have the fixed point property. We also show that if a $C^{*}$-algebra $\mathfrak{A}$ has the fixed point property then for any normal element $a$ of $\mathfrak{A}$, the spectrum $\sigma(a)$ is countable and that the group $C^{*}$-algebra $C^{*}(G)$ has weak normal structure if and only if $G$ is finite.
\end{abstract}

\section{INTRODUCTION}

Let $E$ be a Banach space and $K$ be a weakly compact convex subset of $E$. We say that $K$ has the fpp (=fixed point property) if every nonexpansive mapping $T: K \rightarrow K$ (i.e., $\|T x-T y\| \leq\|x-y\|$ for every $x, y \in K$ ) has a fixed point. The space $E$ has the fpp if every weakly compact convex $K \subseteq E$ has the fpp.

It is well known (Schauder's Theorem) that compact convex nonempty subsets of a Banach space have the fpp. In particular, any Banach space $E$ having the Schur property (i.e., weakly compact subsets of $E$ are norm compact) has the fpp. It is also well-known (Browder's Theorem [6]) that uniformly convex Banach spaces have the fpp. In [14] Kirk, extending Browder's Theorem, showed that a weakly compact convex subset of a Banach space with normal structure has the fpp. In [2] Alspach exhibited a weakly compact convex subset $K$ of the Lebesgue space $L^{1}[0,1]$ and an isometry $T: K \rightarrow K$ without a fixed point, proving, thereby, that the space $L^{1}[0,1]$ does not have the fpp. However, in [19], Maurey, using the techniques of ultraproducts, showed that reflexive subspaces of $L^{1}[0,1]$, as well as the sequence space $c_{0}$, have the fpp.

In this paper we present, in section 3, sufficient conditions and some necessary conditions for the Fourier algebra $A(G)$ and the Fourier-Stieltjes algebra $B(G)$

Received by the editors August 29, 1995 and, in revised form, January 19, 1996.

1991 Mathematics Subject Classification. Primary 43A10, 43A15, 46B20, 47H09, 22D10; Secondary $54 \mathrm{G} 12$.

Key words and phrases. Locally compact groups, Fourier and Fourier-Stieltjes algebras, group algebra, $C^{*}$-algebra, fixed point property, weak-normal structure, uniformly Kadec-Klee property, Radon Nikodym property.

The first author's research was supported by an NSERC grant and the third author's research was supported by TUBA.

(C) 1997 American Mathematical Society 
associated to a locally compact group $G$ to have the fpp. We show that if $G$ is an $[A U]$-group (i.e., the enveloping von Neumann algebra of the group $C^{*}$-algebra $C^{*}(G)$ is atomic) then the spaces $A(G)$ and $B(G)$ have the fpp. In particular, if $G$ is a compact group then $A(G)$ has the fpp (see [16]). Conversely, if $A(G)$ has the fpp then $G$ is compact if $G$ is either a direct product of a compact group and a locally compact abelian group, or a connected $[I N]$-group. We also show that if $A(G)$ has the fpp and $G$ is a $[S I N]$-group then $G$ must contain an open compact normal subgroup. If, in addition, $G$ is discrete then $G$ cannot contain an infinite abelian subgroup; in particular, each element of $G$ must have finite order. In section 4 , we show that if a $C^{*}$-algebra $\mathfrak{A}$ has the fpp then, for any normal element $a$ of $\mathfrak{A}$, the spectrum $\sigma(a)$ of $a$ is countable; consequently, the algebra is scattered. It is also shown that the group $C^{*}$-algebra $C^{*}(G)$ of a locally compact group $G$ has weak-normal structure if and only if $G$ is finite.

\section{Preliminaries}

Let $K$ be a bounded closed convex subset of a Banach space $E$. A point $x$ in $K$ is called a diametral point if

$$
\sup \{\|x-y\|: y \in K\}=\delta(K),
$$

where $\delta(K)$ denotes the diameter of $K$. The set $K$ is said to have normal structure if every nontrivial (i.e., contains at least two points) convex subset $H$ of $K$ contains a nondiametral point of $H$. A Banach space has weak-normal structure if every nontrivial weakly compact convex subset has normal structure. A Banach space having weak-normal structure has the fpp [1].

A Banach space $E$ is said to have the uniformly Kadec-Klee $(=U K K)$ property if for any $\epsilon>0$ there is a $0<\delta<1$ such that whenever $\left(x_{n}\right)$ is a sequence in the unit ball of $E$ converging weakly to $x$ and $\inf \left\{\left\|x_{n}-x_{m}\right\|: n \neq m\right\}>\epsilon$, then $\|x\| \leq \delta$ (see Huff [12]). As is known [8], if $E$ has the $U K K$ property, then $E$ has weak-normal structure and hence the fpp.

A Hausdorff topological space $X$ is dispersed (or scattered) if $X$ does not contain any perfect subset (i.e., a closed subset $A$ of $X$ such that each point of $A$ is an accumulation point of $A$ ).

Now let $G$ be a locally compact group. For the definitions of the Fourier algebra $A(G)$, the Fourier-Stieltjes algebra $B(G)$, the group $C^{*}$-algebra $C^{*}(G)$ and the von Neumann algebra $V N(G)$ we refer the reader to Eymard's paper [9]. The group $G$ is said to be an $[A U]$-group if the von Neumann algebra generated by every continuous unitary representation of $G$ is atomic. It is said to be an $[A R]$-group if the von Neumann algebra $V N(G)$ is atomic. We have the following inclusions

$$
[\text { compact }] \subseteq[A U] \subseteq[A R],
$$

where [compact] denote the class of compact groups, etc. Moreover, these inclusions are proper [23]. The group $G$ is called an $[I N]$-group if there is a compact neighbourhood of the identity $e$ in $G$ which is invariant under inner automorphisms; $G$ is a $[S I N]$-group if there is a base for the neighbourhood system of $e$ consisting of compact sets invariant under inner automorphisms (or equivalently, the left and right uniformities on $G$ are the same). Obviously [compact] $\subseteq[S I N] \subseteq[I N]$ and the inclusions are also proper. Furthermore, all $[I N]$-groups are unimodular(see $[20])$.

For the undefined notations we refer the reader to the book [11]. 


\section{The RNP And the fpp on the predual of Von Neumann Algebras}

We recall that a Banach space $E$ is said to have the Radon-Nikodym property $(=\mathrm{RNP})$ if each closed convex subset $D$ of $E$ is dentable, i.e., for any $\epsilon>0$ there exists an $x$ in $D$ such that $x \notin \overline{\mathrm{co}}\left(D \backslash B_{\epsilon}(x)\right)$, where $B_{\epsilon}(x)=\{y \in X:\|x-y\|<\epsilon\}$ and $\overline{\mathrm{co}} K$ is the closed convex hull of a set $K \subseteq E$. Ample information about this notion can be found in Bourgin's book [5]. We also recall that the dual space of a $C^{*}$-algebra $\mathfrak{A}$ has the RNP if and only if $\mathfrak{A}$ does not contain an isomorphic copy of $l_{1}$ [10, Corollary VII.10] and [5, Corollary 4.1.7]. In general, there exists no connection between the RNP and the fpp, given that the RNP is an "isomorphic property" whereas the fpp is an "isometric property". However, for the preduals of von Neumann algebras we have the following result. See also Lemma 1 in [16].

3.1. Lemma. Let $\mathfrak{M}$ be a von Neumann algebra. If $\mathfrak{M}_{*}$ has the $R N P$, then $\mathfrak{M}_{*}$ has the fpp.

Proof. Let $S$ denote the normal states in $\mathfrak{M}_{*}$ and $\operatorname{ext}(S)$ be the set of its extreme points. As in the proof of Theorem 4 in [7], if for each $f \in \operatorname{ext}(S),\left\{\pi_{f}, \mathfrak{H}_{f}\right\}$ is the representation of $\mathfrak{M}$ induced by the GNS-construction, then $\mathfrak{M}$ is isomorphic to the direct-sum $\sum \oplus \mathcal{B}\left(\mathfrak{H}_{f}\right)$ taken over all $f \in \operatorname{ext}(S)$, where $\mathcal{B}\left(\mathfrak{H}_{f}\right)$ denote the set of all bounded linear operators from the Hilbert space $\mathfrak{H}_{f}$ into itself. It follows from the uniqueness of the predual of a von Neumann algebra that $\mathfrak{M}_{*}$ is linearly isometric to the $l_{1}$-sum $\sum \oplus \mathcal{T}\left(\mathfrak{H}_{f}\right), \quad f \in \operatorname{ext}(S)$, where $\mathcal{T}\left(\mathfrak{H}_{f}\right)$ denotes the space of trace class operators on $\mathfrak{H}_{f}$. Let $\mathfrak{H}$ be the Hilbert space direct summand of $\left\{\mathfrak{H}_{f}, f \in \operatorname{ext}(S)\right\}$. Then the space $\sum \oplus \mathcal{T}\left(\mathfrak{H}_{f}\right)$ can be isometrically embedded into $\mathcal{T}(\mathfrak{H})$. By a result of Lennard [18] $\mathcal{T}(\mathfrak{H})$ has property $U K K$. It follows that $\mathcal{T}(\mathfrak{H})$, and so every subspace of it, has the fpp. Hence $\mathfrak{M}_{*}$ has the fpp.

3.2. Corollary. Let $G$ be a locally compact group. If $C^{*}(G)$ does not contain an isomorphic copy of $l_{1}$, then $B(G)$ has the fixed point property.

Proof. If $C^{*}(G)$ does not contain an isomorphic copy of $l_{1}$ then by Corollary VII.10 in $[10$, p. 96$] B(G)$ has the Radon-Nikodym property. Since $B(G)$ is the predual of the von Neumann algebra $C^{*}(G)^{* *}$ it follow from Lemma 3.1 that $B(G)$ has the fpp.

3.3. Corollary. Let $G$ be a locally compact group.

(1) If $G$ is an $[A U]$-group, then both spaces $B(G)$ and $A(G)$ have the fpp.

(2) If $G$ is an $[A R]$-group, then the space $A(G)$ has the fpp.

Proof. We first observe that, for any locally compact group $G, B(G)=C^{*}(G)^{*}$ and that $A(G)$ is a closed ideal of $B(G)[9]$. Thus $B(G)$ is the predual of the von Neumann algebra $C^{*}(G)^{* *}$. Now assume that $G$ is an $[A U]$-group. Then, by Theorem 4.2 of [23] or Theorem 5 of [4], $B(G)$ has the RNP. Hence, by the preceding lemma, the spaces $B(G)$ and $A(G)$ have the fpp. Now if $G$ is an $[A R]$-group, then, by Theorem 4.1 of [23] or Theorem 4 of [4], $A(G)$ has the RNP. Since $A(G)$ is the predual of the von Neumann algebra $V N(G)[9]$, by the preceding lemma, $A(G)$ has the fpp.

Since the inclusion $[A U] \subseteq[A R]$ is strict, it seems quite possible that there are locally compact groups $G$ for which $A(G)$ has the fpp but $B(G)$ lacks it. However, as we do not know whether the converse of Lemma 3.1 holds or not, we were not able to find a locally compact group $G$ for which $A(G)$ does have the fpp but $B(G)$ 
lacks it. We also remark that if $G$ is a noncompact $[A U]$-group then $B(G)$ does not have the Schur property [17, Theorem 4.5] although it has the RNP and the fpp.

It follows from above that if $G$ is compact, then $A(G)=B(G)$ always has the fixed point property. However, there are non-compact solvable locally compact groups $G$ (for example, when $G$ is the so-called "ax $+\mathrm{b}$ " group= the affine group) such that $V N(G)$ is atomic (see [3]). For such $G, A(G)$ has the fpp by Lemma 3.1. Also, if $G$ is the Fell's group (which is the natural semi-direct product of the $p$-adic numbers with the compact group of $p$-adic units for a fixed prime $p$ ) then $G$ is non-compact, totally disconnected and has countable dual; so it is an $[A U]$-group [23, Remark 4.6]. So $B(G)$ has the fpp by the above corollary.

We now give some necessary conditons for $A(G)$ to have the fpp.

3.4. Lemma. Let $G$ be any locally compact group for which $A(G)$ has the fpp.

(1) If $K$ is any compact normal subgroup of $G$ then $A(G / K)$ also has the fpp.

(2) If $H$ is any open subgroup of $G$ then $A(H)$ also has the fpp.

Proof. (1) By Proposition 3.25 in [9], $A(G / K)$ can be identified isometrically with the subspace of functions in $A(G)$ that are constant on the cosets of $K$. Hence $A(G / K)$ has the fpp.

(2) The map which sends each function $f$ in $A(H)$ to a function which agrees with $f$ on $H$ and zero outside $H$ is a linear isometry from $A(H)$ into $A(G)$ by Proposition 3.21 in [9]. Hence the result follows.

3.5. Theorem. Suppose $G$ is either a direct product of a compact group and a locally compact abelian group, or a connected $[I N]$-group. Then $A(G)$ has the fpp if and only if $G$ is compact.

Proof. One direction follows from Corollary 3.3 (see also [16]). To prove the other direction, first suppose $G=K \times V$, where $K$ is compact and $V$ is a locally compact abelian group. Since $G / K$ is isomorphic to $V, A(G / K)$ and $A(V)$ are isometrically isomorphic. If $A(G)$ has the fpp then, by Lemma 3.4, $A(G / K)$, and hence $A(V)$, have the fpp. But $V$ is abelian, and so, as well known, $A(V)$ is isometrically isomorphic to $L^{1}(\widehat{V})$, where $\widehat{V}$ denotes the dual group of $V$. If $V$ is non-compact then $\widehat{V}$ is non-discrete and so $L^{1}(\widehat{V})$ must contain an isometric copy of $L^{1}[0,1][15$, p. 136], which would contradict that $A(V)$ has the fpp. Hence $V$ must be compact and $G$ is compact.

If $G$ is an $[I N]$-group then by $[20$, diagram 1, p. 698] $G$ contains a compact normal subgroup $K$ such that $G / K$ is a $[S I N]$-group. Hence we may assume $G$ itself is a $[S I N]$-group by Lemma 3.4. Since $G$ is connected, $G$ is the direct product of a compact group and a vector group [20, diagram 2, p. 699]. By the first part of this proof $G$ is compact.

3.6. Proposition. Let $G$ be a $[S I N]$-group. If $A(G)$ has the fpp then $G$ contains an open compact normal subgroup. Furthermore, if $G$ is discrete then $G$ cannot contain an infinite abelian subgroup; in particular, each element of $G$ must have finite order.

Proof. If $G$ is a $[S I N]$-group then $G$ is the discrete extension $(V \times K)^{D}$ of the direct product of a vector group $V$ and a compact group $K$ by a discrete group $D[20$, p. 716$]$. Since a group has a proper open normal subgroup if and only if it has a nontrivial discrete quotient group, this implies that $H:=V \times K$ is an open 
normal subgroup of $G$. Since $A(H)$ also has the fpp Theorem 3.5 shows that $H$ is compact.

If $G$ is discrete and $H$ is an infinite abelian subgroup, then Lemma 3.4 implies $A(H)$ has the fpp. This would contradict Theorem 3.5.

\section{Dispersed Locally COMPaCt SPACES AND The FPP}

Let $X$ be a locally compact (Hausdorff) space. It is well-known that $M(X)=$ $C_{0}(X)^{*}$ has the RNP if and only if $X$ is dispersed [21]. Consequently dispersed locally compact spaces and the fpp are closely connected. In this section we present some results about this connection.

4.1. Theorem. Let $X$ be a locally compact Hausdorff space. If $C_{0}(X)$ has the fpp then $X$ is dispersed.

Proof. Assume that $C_{0}(X)$ has the fpp but $X$ is not scattered. Then $X$ must contain a nonempty compact perfect subset $K$. By Theorem 2 on page 29 in [15], there is a continuous function $\phi$ from $K$ onto [0,1]. By Tietze's Extension Theorem as given in $[11,7.40$, p. 99] $\phi$ has a continuous extension $\tilde{\phi}$ to $X$ that maps $X$ onto $[0,1]$ and has a compact support. It follows that we have a linear isometry from $C[0,1]$ into $C_{00}(X)$, and so into $C_{0}(X)$. The space $C[0,1]$, being universal for separable Banach spaces, contains an isometric copy of the Lebesgue space $L^{1}[0,1]$. As this latter space lacks the fpp, $C_{0}(X)$ lacks it too. This contradiction proves the theorem.

We do not know whether the converse of this theorem is true or not. For the dual space of $C_{0}(X)$, a more satisfactory result is given in Lemma 1 in [16], where it is shown that $C_{0}(X)^{*}$ has the fpp iff $X$ is dispersed.

We now present some corollaries of the above results. If $\mathfrak{A}$ is any $C^{*}$-algebra, for $a \in \mathfrak{A}$, we denote the spectrum of $a$ by $\sigma(a)$.

4.2. Corollary. Let $\mathfrak{A}$ be a $C^{*}$-algebra that has the fpp.

(1) The dual $\mathfrak{B}^{*}$ of every commutative $C^{*}$-subalgebra $\mathfrak{B}$ of $\mathfrak{A}$ has the Schur property.

(2) For any normal element a of $\mathfrak{A}, \sigma(a)$ is countable. In particular, the algebra $\mathfrak{A}$ is scattered.

Proof. (1) Since $\mathfrak{A}$ has the fpp, every commutative $C^{*}$-subalgebra $\mathfrak{B}$ of $\mathfrak{A}$ also has the fpp. As $\mathfrak{B}=C_{0}(X)$ for some locally compact space $X$, Theorem 4.1 shows that $X$ is dispersed. Hence $\mathfrak{B}^{*}$ has the Schur property. See [21].

(2) Let $a$ be a normal element of $\mathfrak{A}$ and $\mathfrak{C}^{*}(a)$ be the closed $C^{*}$-subalgebra of $\mathfrak{A}$ generated by $a$. Then $\mathfrak{C}^{*}(a)$ is an abelian $C^{*}$-subalgebra of $\mathfrak{A}$ and its Gelfand spectrum is $\sigma(a)$. It follows from assertion (1) that $\sigma(a)$ is a dispersed locally compact subset of the complex plane. Since the dispersed subsets of the complex plane are countable by the Cantor-Bendixson Theorem [11, p. 52], the set $\sigma(a)$ is countable. The second statement follows from the local characterization given by Huruya [13], where he proved that a $C^{*}$-algebra is scattered iff the spectrum of each of its self-adjoint element is scattered.

4.3. Corollary. Let $G$ be a locally compact group. Then the $C^{*}$-algebra $C_{0}(G)$ has the fpp if and only if $G$ is discrete. 
Proof. If $C_{0}(G)$ has the fpp then, by Theorem 4.1, $G$ is dispersed, and so it is discrete. The converse follows from Maurey's theorem asserting that $c_{0}$ has the fpp, and its extensions. See [1, p. 108-111].

As another corollary of Theorem 4.1 we have the following result.

4.4. Corollary. A von Neumann algebra $\mathfrak{M}$ has the fpp if and only if $\mathfrak{M}$ is finite dimensional.

Proof. If $\mathfrak{M}$ is infinite dimensional, then every maximal abelian *-subalgebra $\mathfrak{B}$ of $\mathfrak{M}$ must be infinite dimensional. In this case, $\mathfrak{B}$ must be ultraweakly closed. Hence $\mathfrak{B}=\sum_{i \in I} \oplus \mathcal{Z}_{\lambda}$, where each $\mathcal{Z}_{\lambda}=L^{\infty}\left(\Omega_{\lambda}, \mu_{\lambda}\right)$ for some probability space $\left(\Omega_{\lambda}, \mu_{\lambda}\right)$ with $\Omega_{\lambda}=\operatorname{supp}\left(\mu_{\lambda}\right)$ (see $[22$, p. 46]). If $I$ is infinite, then $\mathfrak{B}$ contains an infinite sequence of pairwise orthogonal projections. Hence $\mathfrak{B}$ contains an isometric copy of $l_{\infty}=C(\beta \mathbb{N})$. As $\beta \mathbb{N}$ is not dispersed, by Theorem 4.1, $\mathfrak{M}$ does not have the fpp. If $I$ is finite, then one of $L^{\infty}\left(\Omega_{\lambda}, \mu_{\lambda}\right)$ must be infinite dimensional. Let $L^{\infty}\left(\Omega_{\lambda}, \mu_{\lambda}\right)$ be infinite dimensional and let $\left(E_{n}\right)_{n \in N}$ be a sequence of pairwise disjoint nonnegligible measurable subsets of $\Omega_{\lambda}$. The mapping $T: l_{\infty} \rightarrow L^{\infty}\left(\Omega_{\lambda}, \mu_{\lambda}\right)$ that sends each $a=\left(a_{n}\right)$ to $T(a)=\sum a_{n} \chi_{E_{n}}$ is a linear isometry. It follows that $L^{\infty}\left(\Omega_{\lambda}, \mu_{\lambda}\right)$, and consequently $\mathfrak{M}$, lacks the fpp. Thus, if $\mathfrak{M}$ is infinite dimensional it cannot have the fpp. The converse is trivial.

From the preceding corollary it follows immediately that the von Neumann algebra $V N(G)$ has the fpp if and only if the group $G$ is finite.

Next we consider weak-normal structure on $C^{*}$-algebras.

4.5. Theorem. $A C^{*}$-algebra $\mathfrak{A}$ has weak-normal structure if and only if it is finite dimensional.

Proof. Assume $\mathfrak{A}$ has weak-normal structure. If $\mathfrak{A}$ is an infinite dimensional $C^{*}$ algebra, and $\mathfrak{B}$ is a maximal abelian *-subalgebra of $\mathfrak{A}$, then $\mathfrak{B}$ must be closed and infinite dimensional. Hence $\mathfrak{B}=C_{0}(X)$ for some infinite locally compact $X$. If $\mathfrak{A}$ has weak-normal structure, then $\mathfrak{B}$ also has weak-normal structure and so has the fpp. By Theorem 4.1, $X$ must be dispersed. Let $\Gamma$ be the set of all isolated points of $X$. As $\Gamma$ is dense in $X$ it must be infinite. For each $t \in \Gamma$, the characteristic function $\chi_{t}$ of $t$ is in $C_{0}(X)$. It follows that we have a linear mapping $T: c_{0}(\Gamma) \rightarrow C_{0}(X)$ that sends each $a=\left(a_{n}\right)$ to $T(a)=\sum a_{n} \chi_{t_{n}}$. This mapping is isometric. Consequently, $c_{0}(\Gamma)$ also has weak-normal structure if $\mathfrak{A}$ has. Since it is well known that $c_{0}(\Gamma)$ does not have weak-normal structure we conclude that $\mathfrak{A}$ must be finite dimensional. The converse is trivial.

4.6. Corollary. Let $G$ be a locally compact group. Then $C^{*}(G)$ has weak-normal structure if and only if $G$ is finite.

Proof. If $C^{*}(G)$ has weak-normal structure, then, by the preceding theorem, $C^{*}(G)$ is finite dimensional. It follows that $B(G)=C^{*}(G)^{*}$ is finite dimensional. As $A(G) \subseteq B(G)$ and $A(G)$ is dense in $C_{0}(G)$ with the sup norm topology, $C_{0}(G)$ is finite dimensional. Thus $G$ is finite. The converse is trivial.

We finish the paper with the following questions.

Question 1. Is the converse of Lemma 3.1 true?

Question 2. Is the converse of Theorem 4.1 true?

Question 3. For a compact group $G$, does $C^{*}(G)$ have the fpp? 


\section{REFERENCES}

1. A. Aksoy and M. A. Khamsi, Nonstandard Methods in Fixed Point Theory, Springer-Verlag, New York, 1990. MR 91i:47073

2. D. Alspach, A fixed point free nonexpansive map, Proc. Amer. Math. Soc. 82 (1981), 423-424. MR 82j: 47070

3. L. Baggett and K. Taylor, Groups with completely regular reducible representation, Proc. Amer. Math. Soc. 72 (1978), 596-600. MR 80b:22009

4. A. Bělanger and B.E. Forrest, Geometric properties of coefficient function spaces determined by unitary representations of a locally compact group. (to appear). MR 96f:22005

5. R. Bourgin, Geometric aspects of convex sets with the Radon-Nikodym property, Lecture Notes in Math., vol. 993, Springer-Verlag, New York, 1983. MR 85d:46023

6. F. E. Browder, Nonexpansive nonlinear operators in Banach spaces, Proc. Nat. Acad. Sci. U. S. A. 54 (1965), 1041-1044. MR 32:4574

7. C. H. Chu, A note on scattered $C^{*}$-algebras and the Radon-Nikodym property, J. London Math. Soc. 24 (1981), 533-536. MR 82k:46086

8. D. van Dulst and B. Sims, Fixed points of nonexpansive mappings and Chebyshev centers in Banach spaces with norms of type (KK), Banach space theory and its applications, Bucharest, 1981, Lectures Notes in Math., vol. 991, Springer-Verlag, New York. MR 84i:46027

9. P. Eymard, L'alg̀ebre de Fourier d'un groupe localement compact, Bull. Soc. Math. France 92 (1964), 181-236. MR 37:4208

10. N. Ghousoub, G. Godefroy, B. Maurey and W. Schachermayer, Some topological and geometrical structures in Banach spaces, Memoirs Amer. Math. Soc. 378 (1987). MR 89h:46024

11. E. Hewitt and K. Stromberg, Real and Abstract Analysis, Spring-Verlag, 1969. MR 43:428

12. R. Huff, Banach spaces which are nearly uniformly convex, Rocky Mountain J. Math. 10 (1980), 743-749. MR 82b:46016

13. T. Huruya, A spectral characterization of a class of $C^{*}$-algebra, Sci. Rep. Niigata Univ. Ser. A 15 (1978), 21-24. MR 58:2322

14. W. A. Kirk, A fixed point theorem for mappings which do not increase distances, Amer. Math. Monthly 72 (1965), 1004-1006. MR 32:6436

15. H. E. Lacey, The Isometric Theory of Classical Banach Spaces, Springer-Verlag, New York, 1974. MR 58:12308

16. A. T. Lau and P. F. Mah, Normal structure in dual Banach spaces associated with a locally compact group, Trans. Amer. Math. Soc. 310 (1988), 341-353. MR 89e:43004

17. A. T. Lau and A. Ülger, Some geometric properties on the Fourier and Fourier-Stieltjes algebras of locally compact groups, Arens regularity and related problems, Trans. Amer. Math. Soc. 337 (1993), 321-359. MR 93g:22007

18. C. Lennard, $\mathcal{C}_{1}$ is uniformly Kadec-Klee, Proc. Amer. Math. Soc. 109 (1990), 71-77. MR 90h:46029

19. B. Maurey, Points fixes des contractions sur convexe forme de $L^{1}$, Seminaine d'Analyse Functionnelle 80-81 Ecole Polytechnique.

20. T. W. Palmer, Classes of nonabelian, noncompact, locally compact groups, Rocky Mountain J. of Math. 8 (1973), 683-741. MR 81j:22003

21. A. Pełczyński and Z. Semedani, Spaces of continuous functions III, Studia Math. 18 (1959), 211-222. MR 21:6528

22. S. Sakai, $C^{*}$-algebras and $W^{*}$-algebras, Springer-Verlag, New York, 1971. MR 56:1082

23. K. Taylor, Geometry of the Fourier algebras and locally compact groups with atomic representations, Math. Ann. 262 (1983), 183-190. MR 84h:43020

Department of Mathematical Sciences, University of Alberta, Edmonton, Alberta, Canada, T6G 2G1

E-mail address: tlau@vega.math.ualberta.ca

Department of Mathematical Sciences, Lakehead University, Thunder Bay, Ontario, CANADA, P7B 5E1

E-mail address: pfmah@cs-acad-lan.lakeheadu.ca

Department of Mathematics, Koc University, 80860-Istinye, Istanbul, Turkey

E-mail address: aulger@ku.edu.tr 